\title{
Dynamic scaling behavior at thermal first-order transitions in systems with disordered boundary conditions
}

\author{
Haralambos Panagopoulos, ${ }^{1}$ Andrea Pelissetto, ${ }^{2}$ and Ettore Vicari ${ }^{3}$ \\ ${ }^{1}$ Department of Physics, University of Cyprus, Lefkosia CY-1678, Cyprus \\ ${ }^{2}$ Dipartimento di Fisica dell'Università di Roma "La Sapienza” and INFN, \\ Sezione di Roma I, I-00185 Roma, Italy \\ ${ }^{3}$ Dipartimento di Fisica dell'Università di Pisa and INFN, Largo Pontecorvo 3, I-56127 Pisa, Italy
}

(Received 18 May 2018; published 15 October 2018)

\begin{abstract}
We investigate the dynamic properties of systems at thermal first-order transitions (FOT), when boundary conditions favor one of the two phases. In particular, we study the dynamic scaling properties arising from a slow heating of systems with disordered boundary conditions, starting in the ordered phase and moving across the FOT. As a theoretical laboratory we consider the two-dimensional Potts model. We show that a power-law dynamic scaling emerges, associated with a mixed regime where the two phases are spatially separated. We argue that these features generally apply to systems at FOTs, when boundary conditions favor one of the two phases. In particular, they should be relevant for the experimental search of FOTs of the quark-gluon plasma in heavy-ion collisions.
\end{abstract}

DOI: 10.1103/PhysRevD.98.074507

Understanding finite-size effects at phase transitions is of great phenomenological importance, because it allows us to correctly interpret experiments and numerical investigations of finite-size systems close to the transition point, where thermodynamic quantities develop singularities in the infinite-volume limit. At continuous transitions finitesize scaling (FSS) [1,2] is characterized by universal power laws, with critical exponents that are independent of the geometry and of the boundary conditions, the latter affecting only FSS functions and amplitudes. In this respect first-order transitions (FOTs) are more complicated. Most theoretical studies, see, e.g., Refs. [3-7], have considered cubic $L^{d}$ systems with periodic boundary conditions $(\mathrm{PBC})$, showing that finite-size effects are generally characterized by power laws related to the space dimension of the system; for instance, the correlation-length exponent is $\nu=1 / d$. However, as noted in Refs. [8,9], finite-size effects strongly depend on the geometry, differing significantly in cubic $L^{d}$ and anisotropic geometries. For instance, in $L^{d-1} \times M$ geometries with $M \gg L$, FSS in Ising systems is characterized by exponential laws in $L$ [8]. Recent studies of quantum FOTs have also reported a significant dependence on the boundary conditions $[10,11]$.

In this paper, we investigate the equilibrium and dynamic behaviors at thermal FOTs in finite-size systems with

Published by the American Physical Society under the terms of the Creative Commons Attribution 4.0 International license. Further distribution of this work must maintain attribution to the author(s) and the published article's title, journal citation, and DOI. Funded by SCOAP . boundary conditions favoring one of the two phases, in particular, the disordered phase, such as open boundary conditions (OBC). For this purpose, we consider the twodimensional (2D) Potts model, which is a standard theoretical laboratory to understand issues concerning the statistical behavior at a thermal FOT. We study the dynamic behavior arising from slow variations of the temperature across the FOT. In particular, we consider a slow heating of the system, starting in the ordered phase and moving across the FOT. We show that disordered boundary conditions give rise to an offequilibrium FSS (OFSS) characterized by a timescale increasing as a power of the size $\ell$, i.e., $\tau(\ell) \sim \ell^{2}$, instead of the usual exponentially large timescale emerging at FOTs. We argue that the mechanism driving these dynamic behaviors is quite general, and therefore that they are expected to be developed at generic thermal FOTs when the boundary conditions favor one of the two phases.

Before presenting our results, we discuss the phenomenological relevance of our study. While $\mathrm{PBC}$ are not usually appropriate to describe realistic situations, $\mathrm{OBC}$ are appropriate whenever the disordered phase is somehow favored by the boundaries. Such a situation arises in many physical contexts. One notable example is provided by heavy-ion collision experiments, probing the phase diagram of hadronic matter. At finite temperature $(T)$, theoretical arguments [12] predict the existence of a high- $T$ quark-gluon phase and of a low- $T$ hadronic phase, separated by a FOT line at finite quark chemical potential, ending at an Ising-like transition point. Heavy-ion collision experiments are trying to find evidence for such a FOT line [13-15]; see, in particular, the planned activities discussed in Ref. [16] and references therein. One major problem in 
identifying its signature is the presence of space-time inhomogeneities in the quark-gluon plasma generated in the collisions. The plasma is expected to be confined in a small region with a size of a few femtometers (fm), and to hadronize within a time interval of a few $\mathrm{fm} / c$. In the appropriate region of the phase diagram, as the plasma cools down, the system is expected to cross the FOT line. However, as the size and timescales are finite, there must be a substantial rounding of the FOT singularities, which may conceal its presence. Therefore, for a correct interpretation of the experimental results, it is important to understand the effects of space-time inhomogeneities at FOTs. Since, for the hadronic transition, the high- $T$ and low- $T$ phases are the ordered and the disordered ones, respectively (such correspondence is the opposite of the usual one) [12], the quarkgluon plasma dynamics corresponds to that of a finite-size statistical system, such as the Potts model, that is slowly heated across a FOT. Note also that hadron matter surrounds the quark-gluon plasma; hence, the appropriate boundary conditions in the corresponding statistical system must favor the disordered phase, as is the case for the OBC. The dynamics across the FOT of a finite-size system with OBC should therefore capture some of the important features of the evolution of a confined quark-gluon plasma cooled down across the FOT toward the hadronic low- $T$ phase. This may lead to a better understanding of the signatures of the FOT line in heavy-ion collisions [17].

The 2D $q$-state Potts model provides a theoretical laboratory to study thermal FOTs. It is defined by

$$
Z=\sum_{\left\{s_{\mathbf{x}}\right\}} e^{-\beta H}, \quad H=-\sum_{\langle\mathbf{x y}\rangle} \delta\left(s_{\mathbf{x}}, s_{\mathbf{y}}\right),
$$

where $\beta \equiv 1 / T$, the sum is over the nearest-neighbor sites of an $L \times L$ lattice, the spins $s_{x}$ are integer variables $1 \leq s_{x} \leq q$, and $\delta(a, b)=1$ if $a=b$ and zero otherwise. It undergoes a phase transition $[18,19]$ at $\beta_{c} \equiv 1 / T_{c}=\ln (1+\sqrt{q})$, which is of first order for $q>4$. At $T_{c}$ the infinite-volume energy density $E \equiv$ $\langle H\rangle / L^{2}$ is discontinuous. We define

$$
E_{r} \equiv \Delta_{e}^{-1}\left(E-E_{c}^{-}\right), \quad \Delta_{e} \equiv E_{c}^{+}-E_{c}^{-},
$$

where $E_{c}^{ \pm} \equiv E\left(T_{c}^{ \pm}\right)$[20], so that $E_{r}=0,1$ for $T \rightarrow T_{c}^{-}$and $T \rightarrow T_{c}^{+}$, respectively. The magnetization $M_{k}$,

$$
M_{k}=\left\langle\mu_{k}\right\rangle, \quad \mu_{k} \equiv \frac{1}{V} \sum_{\mathbf{x}} \frac{q \delta\left(s_{\mathbf{x}}, k\right)-1}{q-1},
$$

is also discontinuous, i.e., $\lim _{T \rightarrow T_{c}^{-}} M_{k}=m_{0}>0$ [20].

One can define an equilibrium FSS (EFSS) [3-10] also at FOTs, with scaling laws that are analogous to those holding at continuous transitions [1,2]. This issue has been mostly investigated for cubic $L^{d}$ systems with $\mathrm{PBC}$, finding that the finite-size coexistence region shows a scaling behavior

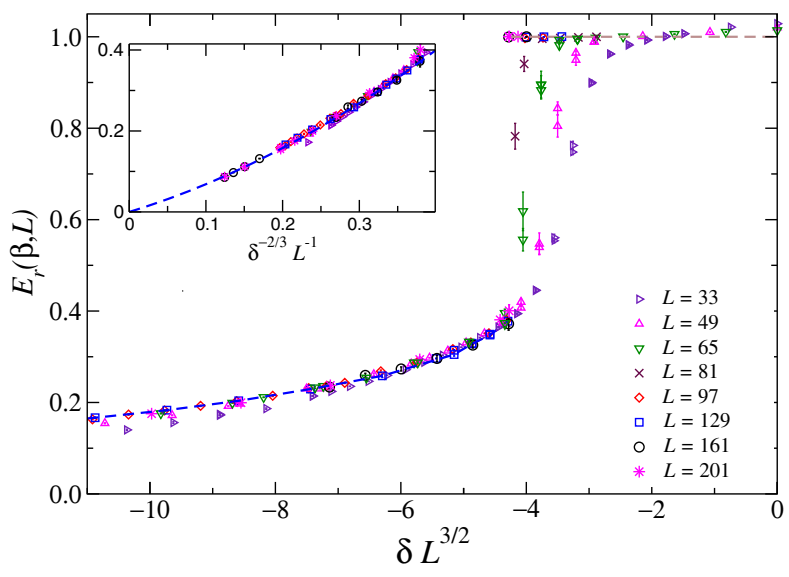

FIG. 1. Equilibrium data for the renormalized energy density $E_{r}$, cf. Eq. (2), for systems with OBC around the FOT point, versus $w \equiv \delta L^{\varepsilon}$ with $\varepsilon=3 / 2$. By increasing the size of the system, the data appear to approach a nontrivial FSS curve (dashed line). The inset shows the data of the low- $T$ region, versus $w^{-1 / \varepsilon}=\delta^{-1 / \varepsilon} L^{-1}$; the dashed line is a fit to $\mathcal{E}_{e}(w) \approx$ $a_{1}|w|^{-1 / \varepsilon}+a_{2}|w|^{-2 / \varepsilon}\left(a_{1} \approx 0.6\right.$ and $\left.a_{2} \approx 1.0\right)$.

in terms of the variable $u \propto \delta L^{d}$ with $\delta \equiv 1-\beta / \beta_{c}$. For instance, the energy density scales as $E_{r}(T, L) \approx \mathcal{E}(u)$. For the 2D Potts model $\mathcal{E}(u)=\left(1+q e^{-u}\right)^{-1}$ [21]. Systems with boundary conditions favoring the high- $T$ phase, such as the $\mathrm{OBC}$, show a more complex behavior, due to surface effects, which give rise to a shift $\delta^{*}(L) \sim 1 / L$ of the transition temperature. Correspondingly, the scaling variable to describe the coexistence regime is $u \propto$ $\left[\delta-\delta^{*}(L)\right] L^{2}$ [23], whose check requires a precise determination of $\delta^{*}(L)$. However, as we shall see, other interesting EFSS regimes emerge.

To investigate these issues, we have performed simulations with $q=20$ (up to $L=200$ ) and $q=10$ (up to $L=512$ ) using OBC [24]. The results appear to scale as

$$
E_{r}(\delta, L) \approx \mathcal{E}_{e}(w), \quad w=\delta L^{\varepsilon},
$$

where $\varepsilon \approx 3 / 2$ with good accuracy (see Fig. 1). This nontrivial scaling is observed for $w<0$ up to an $L$-dependent value $w^{*}(L)$. Since $E_{r}=0$ in the $L \rightarrow \infty$ limit for $\delta=0^{-}$, we expect $\mathcal{E}_{e}(w \rightarrow-\infty) \rightarrow 0$. Moreover, since for $\mathrm{OBC}$ the $L \rightarrow \infty$ limit at fixed $T$ is approached with $1 / L$ corrections, we expect $\mathcal{E}_{e}(w) \approx|w|^{-1 / \varepsilon}$ for $w \ll-1$. This is confirmed by the data for $w \lesssim w^{*}$ (see the inset of Fig. 1). For $w>w^{*}(L)$, the scaling behavior is trivial as $\mathcal{E}_{e}(w)=1$, the high- $T$ value, with $1 / L$ corrections. Analogous results are obtained for other observables and for $q=10$. For $w=w^{*}(L)$ the energy has a two-peak structure (see Fig. 2), and the probabilities of the corresponding configurations are approximately equal. The value $w^{*}(L)$ is therefore related to the shift $\delta^{*}(L)$ of the transition [25]. Note that the scaling in terms of $w$ is not appropriate to describe the region around coexistence, 


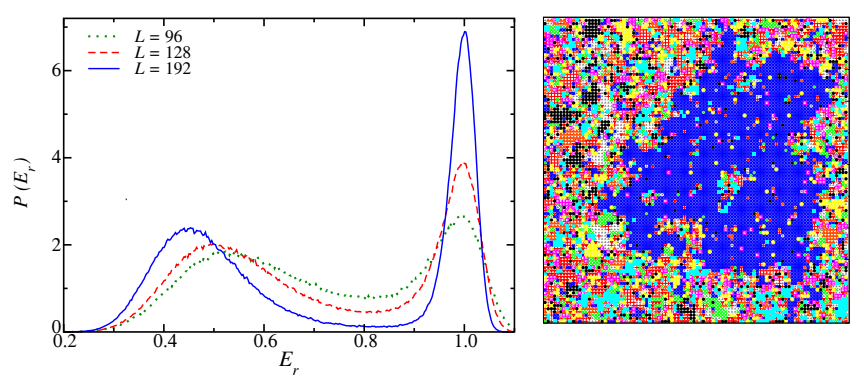

FIG. 2. (Left) Distribution of $E_{r}$ for various values of $L$ at $w=w^{*}$, which is where the specific heat has a maximum (this is very close to the value where the areas below the two peaks are equal). Here $q=10$. (Right) A typical "low-temperature" configuration with $L=192$ at $w=w^{*}$ : the same color and symbol correspond to the same value of the spin.

where the double-peak structure appears. It is expected to describe a low- $T$ regime for $\delta$ strictly smaller than $\delta^{*}(L)$, thus $w<w^{*}$, while it fails when approaching $w^{*}$.

To interpret the scaling in terms of $w$, we note that, for $w<w^{*}(L)$, typical configurations are characterized by a central ordered region surrounded by a disordered ring of volume $V_{+}$, stabilized by the OBC (see the right panel of Fig. 2). Since $E_{r} \approx 1$ in the disordered region and $E_{r} \approx 0$ in the ordered one, its spatial average is related to the size of the disordered region,

$$
E_{r} \approx V_{+} / L^{2}
$$

At fixed $w$, the energy $E_{r}$ is fixed, implying that the scaling limit (4) is appropriate to describe a mixed regime characterized by the simultaneous presence of disordered and ordered regions. This should not be confused with thermodynamic coexistence: for $w<w^{*}(L)$ the free energy has only one minimum (thermodynamically, it represents a low- $T$ state), except in a small interval of $w$ around $w^{*}$, which shrinks as $L$ increases. Therefore, the scaling (4) is appropriate to describe the low- $T$ side of the transition, in an interval $\delta \sim L^{-\varepsilon}$ where a significant part of the volume is disordered. Note that, since $\left|w^{*}(L)\right|$ increases with $L$ [25], this scaling region is restricted to larger and larger values of $|w|$ with increasing $L$. Nevertheless, this regime turns out to be relevant for the dynamic behavior across the FOT (see below).

We now discuss the dynamical behavior of the system, assuming that it is slowly heated across the FOT, starting from the low- $T$ phase. We consider the linear protocol

$$
\delta(t) \equiv 1-\beta(t) / \beta_{c}=t / t_{s}
$$

where $t \in\left[t_{i}<0, t_{f}>0\right]$ is a time variable varying from a negative to a positive value, and $t_{s}$ is the timescale of the process. The value $t=0$ corresponds to $\beta(t)=\beta_{c}$. The dynamics starts from an ordered configuration with $M_{1}=1$, cf. Eq. (3), at $\beta_{i}=\beta_{c}\left[1-\delta\left(t_{i}\right)\right]>\beta_{c}$, so that
$\delta\left(t_{i}\right)<\delta^{*}(L)$ [26]. Then, the system evolves under a heat-bath MC dynamics [24], which corresponds to a purely relaxational dynamics [27]. The time unit is a heat-bath sweep of the whole lattice. The temperature is changed according to Eq. (6) every sweep, incrementing $t$ by one. We repeat this procedure several times, averaging the observables, such as the energy density $E\left(t, t_{s}, L\right)=$ $\langle H\rangle_{t} / V$ and magnetization $M_{1}\left(t, t_{s}, L\right)=\left\langle\mu_{1}\right\rangle_{t}$, over the ensemble of configurations obtained by the off-equilibrium protocol at time $t$.

In systems with $\mathrm{OBC}$ the transition from the low- $T$ to the high- $T$ phase occurs through a mixed-phase regime, which is related to the dynamics of a closed domain wall, with a relatively small (negligible in the large- $L$ limit) thickness, that spatially separates the two phase regions, similarly to the right panel of Fig. 2. For $w \ll w^{*}$, such a domain wall is localized at the boundaries; thus, $E_{r} \approx 0$. With increasing $w$, it moves toward the center of the lattice, until the whole volume is in the high- $T$ phase; thus, $E_{r} \approx 1$. The transition occurs through a free-energy barrier; hence, we expect the system to be out of equilibrium as one moves from one phase to the other.

To identify a scaling regime that describes the dynamic behavior, we must specify appropriate scaling variables. First, we wish to recover the EFSS defined in Eq. (4) in the appropriate limit (see below). Thus, the corresponding dynamic scaling limit should be defined at fixed $r_{1}=\delta(t) L^{\varepsilon}=t L^{\varepsilon} / t_{s}$. The second scaling variable must be $t / \tau(L)$, where $\tau(L)$ is the timescale of the dynamics. The identification of $\tau(L)$ is strictly dependent on the choice of the EFSS variable. At fixed $r_{1}$, the transition region in which the free energy shows a double-peak structure shrinks as $L \rightarrow \infty$. Therefore, the mixed-disordered coexistence region at $\delta^{*}$ is not relevant for the scaling described here. The time interval $\Delta t$ that the system spends in this region is vanishingly small compared to the relevant timescales: $\Delta t L^{\varepsilon} / t_{s} \rightarrow 0$ in the scaling limit. The transition from the ordered to the disordered phase occurs for $r_{1}>w^{*}$, i.e., when the free energy has only the high- $T$ minimum. Under these conditions, we expect $\tau(L) \sim L^{z}$ with $z=2$, which is the expected behavior of the timescale for the shrinking of an ordered domain surrounded by the more stable disordered phase under a purely relaxational dynamics [28]. Therefore, the relevant scaling variables are $r_{1}=\left(t / t_{s}\right) L^{\varepsilon}$ and $r_{2}=t / L^{z}$, or their combinations

$$
s_{1}=t_{s} / L^{\varepsilon+z}, \quad s_{2}=t / t_{s}^{z /(\varepsilon+z)} .
$$

OFSS develops in the limit $t, t_{s}, L \rightarrow \infty$, keeping $s_{1}$ and $s_{2}$ fixed. For example, we expect

$$
E_{r}\left(t, t_{s}, L\right) \equiv \Delta_{e}^{-1}\left[E\left(t, t_{s}, L\right)-E_{c}^{-}\right] \approx \mathcal{E}_{o}\left(s_{1}, s_{2}\right) .
$$

Analogously, $m_{r} \equiv M_{1}\left(t, t_{s}, L\right) / m_{0} \approx \mathcal{M}_{o}\left(s_{1}, s_{2}\right)$. The EFSS of Eq. (4) must be recovered for large values of 

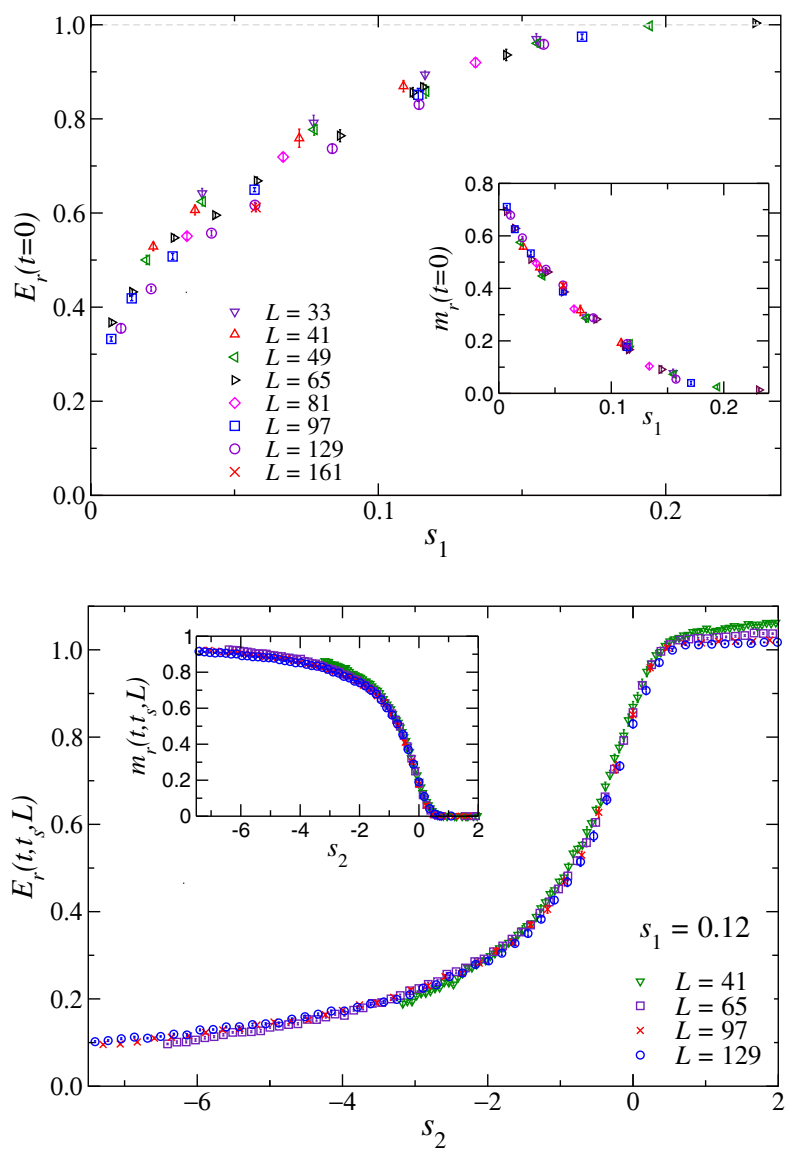

FIG. 3. Plots of $E_{r}$, cf. Eq. (8), and $m_{r} \equiv M_{1} / m_{0}$ (insets) at $t=0$ (top) and fixed $s_{1}=0.12$ (bottom). They support the OFSS predictions.

$s_{1}$ keeping $r_{1}$ fixed, where $\mathcal{E}_{o}\left(s_{1}, s_{1}^{1+z / \varepsilon} r_{1}\right) \approx \mathcal{E}_{e}\left(r_{1}\right)$. The MC results fully support the above OFSS. In particular, Fig. 3 shows that the data at $t=0$, thus $s_{2}=0$, approach a function of $s_{1}=t_{s} / L^{7 / 2}$, and the data at fixed $s_{1} \approx 0.12$ approach a function of $s_{2}=t / t_{s}^{4 / 7}$ (analogous results are obtained for other values of $s_{1}$ ).

It is important to stress the difference between the types of scaling reported above and those discussed in the literature. For instance, for PBC the relevant timescale scales exponentially [22], $\tau(L) \sim \exp (\sigma L)$, as it is related to the tunneling time between the two phases [29]. The different OFSS observed here has been made manifest by the particular choice of the scaling variables, which has allowed us to focus on the emergent mixed regime of systems with OBC. On the other hand, the relevant timescale of the coexistence two-peak region (see Fig. 2) is expected to be much larger, $\tau(L) \sim e^{\alpha L}$, with $\alpha$ related to the corresponding free-energy barrier. Therefore, two different off-equilibrium regimes arise in terms of $t_{s}$. There is an intermediate regime in which $t_{s}$ scales as $L^{\varepsilon+z} \approx L^{7 / 2}$, in which we observe scaling in terms of $s_{1}$ and $s_{2}$. On the other hand, for exponentially large $t_{s} \sim e^{\alpha L}$, one should observe a different scaling appropriate to the dynamics in the coexistence (two-peak) region.

Our results show that FSS behaviors at FOTs strongly depend on the boundary conditions. In particular, in systems with boundary conditions that favor one of the two phases, there exists a scaling regime characterized by the presence of both phases in separate central and boundary spatial regions. Of course, this dynamic regime is expected to emerge also in systems with different geometries, such as elongated or slablike systems; numerical checks support this expectation. Starting from this mixed regime, if one slowly heats the system across the transition, one may define a dynamical scaling with a characteristic time $\tau(L)$ that scales as $L^{2}$. We argue that the main features of the mechanism driving these off-equilibrium behaviors are quite general; thus, they are expected to be developed by generic systems at thermal FOTs when boundary conditions favor one of the two phases. Moreover, we expect that they also emerge for other types of dynamics beside the purely relaxational ones, e.g., in the presence of conservation laws. We note that power-law OFSS are generally observed at continuous transitions [30-32]. Our study shows that the main distinguishing feature of the OFSS across a FOT is related to the existence of a mixed regime, where the two phases are spatially separated.

Analogous off-equilibrium scaling scenarios are expected to also arise at quantum FOT of many-body systems whose boundary conditions favor one of the two phases. In particular, equilibrium behaviors analogous to that observed in 2D Potts models, cf. Eq. (4), also emerge in 1D quantum Ising chains along their quantum FOT line driven by an external longitudinal field [33]. Similarly to Potts models, they are driven by the dynamics of a domain wall (kinks). However, the corresponding exponent turns out to be $\varepsilon=2$ (as inferred by theoretical arguments and supported by numerical results). Using arguments based on the standard quantum-to-classical mapping, we expect that a dynamic scenario analogous to that observed at the FOT of 2D Potts models develops also at the FOTs of the 2D Ising models defined in slab geometries, when a magnetic field is slowly driven across the FOT line in the low-temperature phase. However, the value of the exponent $\varepsilon$ of the corresponding anomalous EFSS must be $\varepsilon=2$. Apart from that, the dynamic behavior arising from slow variations of the driving parameter is expected to develop an analogous power-law scaling regime, instead of the usual exponential timescales emerging at FOTs (in this respect, the value of $\varepsilon$ is not crucial, because any value leads to a power law).

Dynamic OFSS behaviors at FOTs in the presence of boundary conditions favoring one of the two phases are of experimental interest. They may be exploited to gain evidence of a FOT in experiments with finite systems in off-equilibrium conditions. For example, they are relevant for heavy-ion experiments [16] searching for evidence of FOTs in the hadron phase diagram [12]. Our study suggests 
that a dynamic mechanism analogous to that observed in Potts models drives the quark-gluon plasma when it cools down across the FOT line, implying a scaling behavior on timescales $\tau \sim \ell^{2}$, where $\ell$ is the size of quarkgluon plasma, a few fm. Note that, since the cooling is relatively fast-typical times should be of the order of a few $\mathrm{fm} / \mathrm{c}$ - this is probably the only scaling behavior that can be observed in practice. As already noted, power-law OFSS behaviors also characterize continuous transitions; however, the key feature of the crossing of a FOT line should be that it occurs through a mixed regime, where the hadronic and quark-gluon phases are spatially separated.
[1] M. N. Barber, Finite-Size Scaling, in Phase Transitions and Critical Phenomena, edited by C. Domb and J. L. Lebowitz (Academic Press, New York, 1983), Vol. 8.

[2] Finite Size Scaling and Numerical Simulations of Statistical Systems, edited by V. Privman (World Scientific, Singapore, 1990).

[3] B. Nienhuis and M. Nauenberg, First-Order Phase Transitions in Renormalization-Group Theory, Phys. Rev. Lett. 35, 477 (1975).

[4] M. E. Fisher and A. N. Berker, Scaling for first-order phase transitions in thermodynamic and finite systems, Phys. Rev. B 26, 2507 (1982).

[5] M. S. S. Challa, D. P. Landau, and K. Binder, Finite-size effects at temperature-driven first-order transitions, Phys. Rev. B 34, 1841 (1986).

[6] C. Borgs and R. Kotecky, A rigorous theory of finite-size scaling at first-order phase transitions, J. Stat. Phys. 61, 79 (1990).

[7] K. Vollmayr, J. D. Reger, M. Scheucher, and K. Binder, Finite-size effects at thermally-driven first order phase transitions: A phenomenological theory of the order parameter distribution, Z. Phys. B 91, 113 (1993).

[8] V. Privman and M. E. Fisher, Finite-size effects at first-order transitions, J. Stat. Phys. 33, 385 (1983).

[9] M. E. Fisher and V. Privman, First-order transitions breaking $\mathrm{O}(n)$ symmetry: Finite-size scaling, Phys. Rev. B 32, 447 (1985).

[10] M. Campostrini, J. Nespolo, A. Pelissetto, and E. Vicari, Finite-Size Scaling at First-Order Quantum Transitions, Phys. Rev. Lett. 113, 070402 (2014); Finite-size scaling at first-order quantum transitions of quantum Potts chains, Phys. Rev. E 91, 052103 (2015).

[11] M. Campostrini, A. Pelissetto, and E. Vicari, Quantum transitions driven by one-bond defects in quantum Ising rings, Phys. Rev. E 91, 042123 (2015); Quantum Ising chains with boundary terms, J. Stat. Mech. (2015) P11015.

[12] K. Rajagopal and F. Wilczek, in At the Frontier of Particle Physics/Handbook of $Q C D$, edited by M. Shifman (World Scientific, Singapore, 2001).

[13] J. D. Bjorken, Highly relativistic nucleus-nucleus collisions: The central rapidity region, Phys. Rev. D 27, 140 (1983).

[14] S. A. Bass, M. Gyulassy, H. Stöcker, and W. Greiner, Signatures of Quark-Gluon-Plasma formation in high energy heavy-ion collisions: A critical review, J. Phys. G 25, R1 (1999).

[15] T. Hirano, P. Huovinen, K. Murase, and Y. Nara, Integrated dynamical approach to relativistic heavy ion collisions, Prog. Part. Nucl. Phys. 70, 108 (2013).
[16] H. Petersen, Beam energy scan theory: Status and open questions, Nucl. Phys. A967, 145 (2017).

[17] We are aware of only one related study: J. Steinheimer and J. Randrup, Spinodal Amplification of Density Fluctuations in Fluid-Dynamical Simulations of Relativistic Nuclear Collisions, Phys. Rev. Lett. 109, 212301 (2012). This is based on a transport model encoding some of the expected features at FOTs related to spinodal decomposition. In this paper we develop scaling arguments that do not rely on spinodal-like approximations.

[18] R. J. Baxter, Exactly Solved Models in Statistical Mechanics (Academic Press, New York, 1982).

[19] F. Y. Wu, The Potts model, Rev. Mod. Phys. 54, 235 (1982).

[20] The $q=20$ Potts model has a FOT at $T_{c}=0.58835 \ldots$, where $E\left(T_{c}^{+}\right)=-0.62653 \ldots, \quad E\left(T_{c}^{-}\right)=-1.82058 \ldots$, $\beta_{c} \kappa=0.18549 \ldots$ The magnetization is discontinuous: $\lim _{T \rightarrow T_{c}^{-}} \lim _{h_{k} \rightarrow 0} \lim _{V \rightarrow \infty} M_{k} \equiv m_{0}=0.941175 \ldots \quad$ in the presence of a magnetic field along the $k$ state, while it vanishes for $T>T_{c}$. See Refs. [18,19] and C. Borgs and W. Janke, An explicit formula for the interface tension of the 2D Potts model, J. Phys. I (France) 2, 2011 (1992); A. Billoire, T. Neuhaus, and B.A. Berg, A determination of interface free energies, Nucl. Phys. B413, 795 (1994); A. Tröster and K. Binder, Microcanonical determination of the interface tension of flat and curved interfaces from Monte Carlo simulations, J. Phys. Condens. Matter 24, 284107 (2012).

[21] This is obtained by expressing the partition function of mixed states around $T_{c}$ as a sum of two exponential functions related to the free energies of the pure high- $T$ and low- $T$ phases $[5,6,22]$, neglecting exponentially suppressed contributions related to the interface. The scaling variable turns out to be $u=\Delta_{e} \beta_{c} \delta L^{2}$.

[22] A. Pelissetto and E. Vicari, Dynamic Off-Equilibrium Transition in Systems Slowly Driven across Thermal First-Order Transitions, Phys. Rev. Lett. 118, 030602 (2017).

[23] V. Privman and J. Rudnick, Nonsymmetric first-order transitions: Finite-size scaling and tests for infinite-range models, J. Stat. Phys. 60, 551 (1990).

[24] We use a standard heat-bath algorithm, which updates a single site variable by generating a new spin $s_{x}{ }^{\prime}$ at $\boldsymbol{x}$ with probability $\sim e^{-H\left(s_{x}{ }^{\prime}\right) / T}$ independent of the old spin.

[25] The surface-induced shift [23] $\delta^{*}(L) \sim 1 / L$ implies that $w^{*}(L) \sim L^{1 / 2}$. Our data around the two-peak region show a weak $L$ dependence (in particular, for $q=20$ ); however, they are not sufficient to evidence the $L^{1 / 2}$ behavior. Its careful check requires larger systems, which can only be 
studied using multicanonical algorithms [B. A. Berg and T. Neuhaus, Multicanonical ensemble: A new approach to simulate first-order phase transitions, Phys. Rev. Lett. 68, 9 (1992)]. Since our study does not focus on the dynamics appropriate to describe the two-peak region, we have not pursued this analysis further.

[26] The resulting asymptotic OFSS around $\delta(t)=0$ does not depend on the initial finite value of $\delta\left(t_{i}\right)<\delta^{*}(L)$, in the low- $T$ region. It does not depend on the final value $\delta_{f}>0$ either.

[27] P. C. Hohenberg and B. I. Halperin, Theory of dynamic critical phenomena, Rev. Mod. Phys. 49, 435 (1977).

[28] A. J. Bray, Theory of phase-ordering kinetics, Adv. Phys. 43, 357 (1994).

[29] Using PBC the tunneling between the two phases occurs by means of the generation of striplike configurations with two interfaces, whose probability is suppressed as $\exp (-\sigma L)$, where $\sigma=2 \beta_{c} \kappa$ and $\kappa$ is the interface tension.

[30] S. Gong, F. Zhong, X. Huang, and S. Fan, Finitetime scaling via linear driving, New J. Phys. 12, 043036 (2010).

[31] A. Chandran, A. Erez, S. S. Gubser, and S. L. Sondhi, Kibble-Zurek problem: Universality and the scaling limit, Phys. Rev. B 86, 064304 (2012).

[32] A. Pelissetto, D. Rossini, and E. Vicari, Off-equilibrium dynamics driven by localized time-dependent perturbations at quantum phase transitions, Phys. Rev. B 97, 094414 (2018).

[33] A. Pelissetto, D. Rossini, and E. Vicari, Finite-size scaling at first-order quantum transitions when boundary conditions favor one of the two phases, Phys. Rev. E 98, 032124 (2018). 